\title{
Análisis Molecular de las Mutaciones 2299delG y C759F en Individuos Colombianos con Retinitis Pigmentosa e Hipoacusia Neurosensorial
}

\author{
Molecular analyses of 2299delG and C759F mutations in Colombian \\ Retinitis Pigmentosa and Sensorineural hearing loss affected individuals \\ Greizy López ${ }^{1}$, Nancy Gelvez ${ }^{2}$, Luisa Fernanda Urrego ${ }^{3}$, Silvia Florez ${ }^{4}$ David Medina $^{4}$, Vicente Rodríguez \\ Marta lucía Tamayo ${ }^{2}, \stackrel{4}{ }$.
}

\section{Resumen}

Objetivo: Determinar la presencia de las mutaciones 2299delG y C759F en 37 individuos colombianos no relacionados que presentan RP e hipoacusia neurosensorial. Materiales y métodos: análisis de secuencia directa del exón 13 del gen $U S H 2 A$ en todos los individuos seleccionados para el estudio. Resultados: la mutación 2299delG fue observada únicamente en individuos con Síndrome de Usher tipo II, mientras que la mutación C759F, no fue identificada en los individuos del estudio.

Palabras clave: síndrome de Usher, trastornos sordoceguera, pérdida auditiva sensorineural, retinitis pigmentosa, mutación.

\begin{abstract}
Objective: Presence of 2299delG and C759F mutations was screened in 37 Colom $\neg$ bian nonrelated individuals presenting RP and sensorineural deafness. Materials and methods: Direct sequence analysis of exon 13 of USH2A gene was performed in all individuals selected for the study. Results: 2299delG mutation was found only related to Usher syndrome type II, and C759F mutation was not found in any of the selected individuals.
\end{abstract}

Keywords: Usher Syndromes, Deaf-Blind Disorders, Sensorineural Hearing Loss, Retinitis Pigmentosa, Mutation.

\footnotetext{
Escuela de Ciencias de la Salud, Grupo BIOINNOVA, Universidad Nacional Abierta y a Distancia - UNAD.

${ }^{2}$ Instituto de Genética Humana, Universidad Javeriana, Bogotá, Colombia.

${ }^{3}$ Universidad del Rosario.

${ }^{4}$ Fundación Oftalmológica Nacional, Bogotá, Colombia.

${ }^{5}$ Departamento de ORL, Hospital San Ignacio, Universidad Javeriana, Bogotá, Colombia.

Correspondencia: mtamayo@javeriana.edu.co
} 


\section{Introducción}

El Síndrome de Usher (USH) es una entidad de herencia autosómica recesiva, que se presenta con hipoacusia neurosensorial, retinitis pigmentosa progresiva y, en algunos casos, disfunción vestibular. El Síndrome es la causa más frecuente de sordo-ceguera en el mundo, y constituye el $6 \%$ de la población congénitamente sorda y el $18 \%$ de toda la población con RP (1); la prevalencia del USH está en un rango de 3.5 a 6.2 casos de cada 100,000 habitantes (2); su frecuencia en Estados Unidos es alrededor de 5/100,000 (1); en Escandinavia de 3.0/100,000 (3) y en Colombia, de 3,2/100.000 habitantes y constituye el 9,6\% de la población sorda y el $10 \%$ de la población ciega (4-6). Clínicamente, el USH se divide en tres tipos: el tipo I (USH1) se caracteriza por sordera profunda congénita, ausencia de respuesta vestibular y aparición de RP progresiva en la primera o principios de la segunda década de vida; el tipo II (USH2) por sordera congénita de moderada a severa, respuesta vestibular normal, con inicio de RP progresiva en la segunda década de vida; y, el tipo III (USH3), por pérdida auditiva progresiva, respuesta vestibular variable y aparición de RP en edad variable (7).

El USH presenta una significativa heterogeneidad genética: a cada uno de los tres tipos clínicos del síndrome le corresponde uno o más subtipos genéticos. Hasta el momento, se han identificado 14 loci asociados con el USH: seis responsables del fenotipo USH1 (USH1B-K), cuatro del USH2 (USH2A-D) y uno del fenotipo USH3 (USH3A). De ellos, han sido identificados nueve genes responsables: $M Y O 7 A$ para USH1B, USH1C para USH1C, CDH23 para USH1D, PCDH15 para USH1F, SANS para USH1G, y CIB2 para USH1J (8-13); USH2A para USH2A, $V L G R 1 b$ para USH2C, WHRN para USH2D y US$H 3 A$ para USH3 (14-18). De todos los anteriormente mencionados, el primer gen identificado fue el USH2A (19), el cual codifica para la proteína Usherina $(20,21)$.

Algunos autores han reportado que el USH2 es la forma más común de Síndrome de Usher, ya que cuenta para casi la mitad de los casos reportados (22-24), aunque en Colombia se observa una frecuencia muy similar entre el USH1 y USH2. Algunos estudios en población europea y americana han reportado también que entre 74 al 83\% de los casos USH2, corresponden al subtipo USH2A $(16,25)$. Inicialmente se describió que el gen $U S H 2 A$ constaba de 21 exones, pero posteriormente se definió que existe una segunda isoforma que comprende 72 exones adicionales a los ya conocidos (26).

Un gran número de mutaciones han sido identificadas en el gen $U S H 2 A$, pero la más prevalente es la $2299 \mathrm{delG}(14,16,27-31)$. Esta mutación ha sido identificada en el $25 \%$ de la población española con USH2 (27) y es responsable tanto del fenotipo de USH tipo II, como USH atípico (32). Existe evidencia que indica que esta mutación tuvo su origen en la población del sur de Europa (33). Otro cambio frecuente en el gen USH2A, es la mutación C759F, la cual es responsable del fenotipo USH2 $(28,34)$ y RP sin sordera (35). Ambas mutaciones están localizadas en el exón 13, una región que representa sólo el 6.4\% de la región codificante de este gen (36). Dada la prevalencia de estas dos mutaciones y la variabilidad fenotípica que presentan, el objetivo de este trabajo fue identificar estos cambios en individuos colombianos con asociación de RP e hipoacusia neurosensorial congénita.

\section{Materiales y métodos Población objeto de estudio}

La población objeto de estudio estuvo conformada por 37 individuos con RP e hipoacusia neurosensorial, que fueron seleccionados en tamizajes realizados por el Instituto de Genética Humana de la Universidad Javeriana y la Fundación Oftalmológica $\mathrm{Na}$ cional (FUNDONAL) en 11 ciudades del país, en institutos para ciegos y sordos. Para ello se realizaron exámenes de fondo de ojo y audiometría. A los individuos seleccionados se les tomaron muestras de $7 \mathrm{~mL}$ de sangre periférica por punción venosa, previa firma del consentimiento informado. El diagnóstico clínico se realizó con base en la historia clínica completa de los individuos afectados, la evaluación del mayor 
número posible de familiares, la elaboración del árbol genealógico y exámenes diagnósticos de electrorretinograma (ERG), campimetría, angiografía, fotos de fondo de ojo y audiometría. Este estudio fue aprobado por el Comité de Ética de la Pontificia Universidad Javeriana.

\section{Pruebas moleculares}

El ADN de las muestras fue extraído por medio de la técnica fenol-cloroformo. Posteriormente se realizó el análisis de secuenciación del exón 13 del gen USH2A en todos los individuos seleccionados para el estudio. El procedimiento utilizado para la secuenciación fue el siguiente: una vez obtenido el producto de PCR (Polymerase Chain Reaction), se purificó y se realizó la reacción de secuenciación con $2 \mu \mathrm{l}$ de mezcla de reacción, $2 \mu \mathrm{l}$ de solución tampón $5 \mathrm{X}, 1 \mu \mathrm{l}$ de iniciador y $5 \mu \mathrm{l}$ de producto purificado. Las condiciones de temperatura fueron: $95{ }^{\circ} \mathrm{C}$ por 10 segundos, $50{ }^{\circ} \mathrm{C}$ por 5 segundos y 60 ${ }^{\circ} \mathrm{C}$ por 4 minutos, durante 35 ciclos. Se realizó la precipitación de la reacción de secuenciación por el método de etanol. Los análisis de secuenciación se realizaron en el ABI-PRISM 3100-AvantTM con el kit de secuenciación BigDye terminatorTM, versión 3.1. Finalmente, se analizaron las secuencias con el software DNAstarTM. La secuencia consenso utilizada para el gen USH2A se obtuvo de: http://www. ensembl.org/Homo_sapiens/Transcript/Summary ?db=core;g=ENSG00000042781; r=1:215796236216596738;t=ENST00000366942.

\section{Resultados}

Para el estudio fueron seleccionados en total 37 individuos no relacionados distribuidos en tres grupos de la siguiente forma: 1) 26 individuos no relacionados con síndrome de Usher tipo II, 2) cuatro individuos no relacionados con fenotipos similares a Síndrome de Usher pero con un mecanismo de herencia autosómico dominante o presencia de individuos con RP sin sordera e individuos con sordera sin RP dentro de la misma familia, y 3) siete individuos no relacionados con RP e hipoacusia leve no diagnosticados como Síndrome de Usher. Se realizó el análisis de se- cuenciación automática del exón 13 del gen USH $2 A$ en las muestras de los 37 individuos seleccionados.

Los resultados del análisis de secuenciación se observan en la Tabla 1. En total, el 18.9\% de la población estudiada, presenta la mutación $2299 \mathrm{delG}$, ya sea en estado homo o heterocigoto (Figura 1) y ninguno presenta la mutación C759F. De la población con diagnóstico de Síndrome de Usher tipo II, cerca del $27 \%$ de los propósitos porta la mutación 2299delG, ya sea en estado homocigoto o heterocigoto.

Tabla 1. Resultado del análisis de secuenciación automática en la población analizada.

\begin{tabular}{|l|c|l|}
\hline FENOTIPO & $\begin{array}{c}\text { Número de } \\
\text { individuos }\end{array}$ & \multicolumn{1}{|c|}{$\begin{array}{c}\text { Resultado } \\
\text { Secuencia }\end{array}$} \\
\hline $\begin{array}{l}\text { Síndrome de } \\
\text { Usher tipo II }\end{array}$ & 1 & $\begin{array}{l}\text { 2299delG en estado } \\
\text { homocigoto }\end{array}$ \\
\hline Similar a USH & 19 & $\begin{array}{l}\text { 2299delG en estado } \\
\text { heterocigoto }\end{array}$ \\
\hline RP con HNS leve & 7 & Ningún cambio identificado \\
\hline TOTAL & 37 & Ningún cambio identificado \\
\hline
\end{tabular}
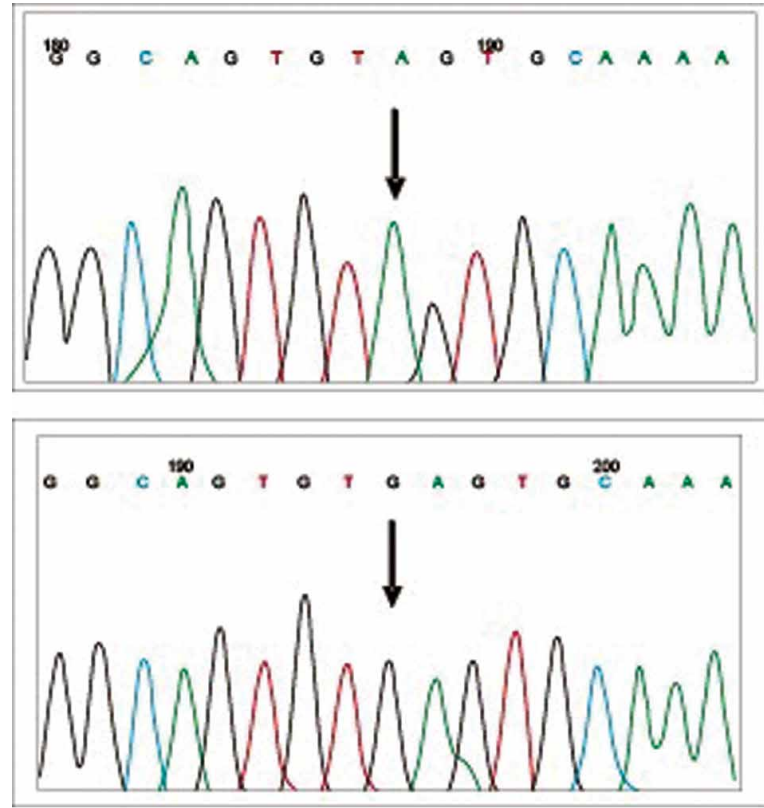

Figura 1. Secuencia del exón 13 del gen $U S H 2 A$. a) Mutación 2299delG en estado homocigoto. b) Secuencia sin la mutación. 
El 3.8\% de los individuos con Síndrome de Usher tipo II, presentan la mutación 2299delG en estado homocigoto, mientras que el $23.1 \%$ la presenta en estado heterocigoto. La frecuencia alélica de esta mutación en la población estudiada con Síndrome de Usher tipo II, es de $15.4 \%$ (Tabla 2).

Tabla 2. Frecuencia alélica de la mutación 2299delG.

\begin{tabular}{lccc|} 
FENOTIPO & $\begin{array}{c}\text { Alelos } \\
\text { 2299delG }\end{array}$ & $\begin{array}{c}\text { Total Alelos } \\
\text { Analizados }\end{array}$ & $\begin{array}{c}\text { Frecuencia } \\
\text { Alélica }\end{array}$ \\
\hline $\begin{array}{l}\text { Síndrome de } \\
\text { Usher tipo II }\end{array}$ & 8 & 52 & $15.4 \%$ \\
\hline Similar a USH & 0 & 8 & 0 \\
$\begin{array}{l}\text { RP con HNS } \\
\text { leve }\end{array}$ & 0 & 14 & 0 \\
\hline
\end{tabular}

\section{Discusión}

La frecuencia del USH en Colombia ha sido estimada en 3,2/100.000 habitantes y constituye el $9,6 \%$ de la población sorda y el $10 \%$ de la población ciega (4-6). Entre la población afectada con USH, casi el $40 \%$ corresponde al tipo II de la enfermedad (datos de los autores), y la necesidad de diagnosticar molecularmente estos individuos, ha incrementado el interés hacia la realización de pruebas confiables para detectar las mutaciones propias de la población colombiana, lo cual será de vital importancia en el momento de realizar un diagnóstico temprano e iniciar un proceso de rehabilitación.

La mutación 2299delG es la mutación más frecuente en individuos con USH2 en el mundo y su frecuencia está estimada en un $25 \%$ en la población española (27). En el presente estudio, se identificó la mutación $2299 \mathrm{deG}$ en un $18.9 \%$ de la población analizada y en un $27 \%$ de los individuos diagnosticados con USH2. Los individuos con la mutación 2299delG tanto en estado homocigoto como heterocigoto, presentaron un fenotipo muy similar y homogéneo de USH típico. En cuanto a los individuos que presentan la mutación en estado heterocigoto, muy seguramente se trata de heterocigotos compuestos, en quienes hasta el momento no se ha identificado la otra mutación causante. Se observaron algunos casos en los que la hipoacusia se manifestó de forma más severa que en otros, lo cual puede ser causado por la presencia de una segunda mutación más agresiva en algunos de los heterocigotos compuestos. Hasta el momento, no se ha identificado esta mutación en pacientes con RP e hipoacusia leve, ni en pacientes con fenotipo similar a USH en Colombia.

La mutación C759F, consiste en una transversión de una Guanina por una Timina que conduce a un cambio de una Cisteína por una Fenilalanina en el residuo 759, y constituye otro cambio frecuente en el gen $U S H 2 A$ en España $(36,37)$. En este estudio, la mutación C759F no fue identificada en ninguno de los individuos de la población analizada, contrario a lo que se esperaba, lo que indica que la población colombiana no puede, en todos los casos, ser comparable con la población española. Estos resultados sugieren que la población colombiana podría tener mutaciones propias; debido, tal vez, a diferentes rutas de migración, el gran bagaje indígena o incluso, mutaciones de tipo efecto fundador. Este mismo fenómeno se ha reportado en otras poblaciones, como es el caso de Japón, en donde existe una marcada diferencia entre su espectro mutacional y el de otras poblaciones $(38,39)$. La población colombiana podría estar mostrando un comportamiento similar al de otras poblaciones como la china o la finlandesa $(40,41)$.

Se conoce que mutaciones en el gen USH $2 A$ son responsables de un amplio espectro de fenotipos y, en especial, las mutaciones 2299delG y C759F, que se han visto asociadas a fenotipos tanto de Síndrome de Usher tipo II, Síndrome de Usher atípico y RP aislada. En los reportes se ha descrito como Síndrome de Usher atípico aquellos individuos que presentan RP e hipoacusia neurosensorial de herencia autosómica recesiva, pero similar al fenotipo del USH3, es decir, con progresión de la hipoacusia $(32,36)$. En el presente estudio, los individuos reportados con un fenotipo similar a USH, son 
individuos que, si se analizan de forma particular, podrían ser diagnosticados como USH tipo II, pero que al analizar el conjunto familiar, se hace evidente un patrón de herencia autosómico dominante que no coincide con los parámetros de diagnóstico del síndrome. En otras de las familias, se observan individuos con lo que parecería ser USH, pero con hermanos con RP aislada y otros con hipoacusia aislada; por lo tanto, no se puede definir en ellos el diagnóstico clínico de USH atípico hasta que se logre identificar los genes responsables.

En conclusión, este estudio muestra que el 27\% de la población colombiana con diagnóstico clínico de Síndrome de Usher tipo II presenta la mutación 2299delG, y esta frecuencia es muy similar a la reportada en Espańa, que es del 25\% (27). La mutación $\mathrm{C} 759 \mathrm{~F}$, no ha sido identificada hasta el momento en individuos con Síndrome de Usher tipo II. Para el futuro, sería importante realizar el mismo tamizaje mutacional en individuos con RP aislada, para poder establecer la frecuencia de las mutaciones en esta población.

\section{Agradecimientos}

Esta investigación fue financiada por COLCIENCIAS con el proyecto "LIGAMIENTO GENÉTICO Y ESTUDIOS MUTACIONALES EN 10 FAMILIAS COLOMBIANAS CON SÍNDROME DE USHER", código no. 1203-04-11732, contrato no. 141-2002 y por el Instituto de Genética Humana de la Universidad Javeriana en Bogotá. Proyecto No 1045 titulado "Ligamiento génico y estudios mutacionales en 10 familias colombianas con síndrome de Usher". Agradecemos al Hospital Universitario San Ignacio por los exámenes audiométricos; a la Fundación Oftalmológica Nacional y a la Clínica Horus por los exámenes oftalmológicos. Al Boys Town Nacional Research Hospital en Omaha, NE, USA. Un especial reconocimiento a los pacientes y sus familiares que gentilmente aceptaron participar en este estudio, así como a todos los institutos de y para nińos ciegos y sordos del país.

\section{Referencias}

1. Boughman JA, Vernon M, Shaver KA. Usher syndrome: definition and estimate of prevalence from two high-risk populations. J Chronic Dis. 1983;36(8):595-603.

2. Keats BJ, Corey DP. The usher syndromes. Am J Med Genet. 1999 Sep 24;89(3):158-66.

3. Hallgren B. Retinitis pigmentosa combined with congenital deafness; with vestibulo-cerebellar ataxia and mental abnormality in a proportion of cases: A clinical and genetico-statistical study. Acta Psychiatr Scand Suppl. 1959;34(138):1-101.

4. Tamayo ML, Bernal JE, Tamayo GE, Frias JL, Alvira G, Vergara $\mathrm{O}$, et al. Usher syndrome: results of a screening program in Colombia. Clin Genet. 1991 Oct;40(4):304-11.

5. Tamayo ML, Bernal JE, Tamayo GE, Frias JL. Study of the etiology of deafness in an institutionalized population in Colombia. Am J Med Genet. 1992 Nov 1;44(4):405-8.

6. Tamayo ML, Maldonado C, Plaza SL, Alvira GM, Tamayo GE, Zambrano M, et al. Neuroradiology and clinical aspects of Usher syndrome. Clin Genet. 1996 Sep;50(3):126-32.

7. Pakarinen L, Tuppurainen K, Laippala P, Mantyjarvi M, Puhakka H. The ophthalmological course of Usher syndrome type III. Int Ophthalmol. 1995;19(5):307-11.

8. Kimberling WJ, Moller CG, Davenport S, Priluck IA, Beighton PH, Greenberg J, et al. Linkage of Usher syndrome type I gene (USH1B) to the long arm of chromosome 11. Genomics. 1992 Dec;14(4):988-94.

9. Smith RJ, Lee EC, Kimberling WJ, Daiger SP, Pelias MZ, Keats BJ, et al. Localization of two genes for Usher syndrome type I to chromosome 11. Genomics. 1992 Dec;14(4):995-1002.

10. Bork JM, Peters LM, Riazuddin S, Bernstein SL, Ahmed ZM, Ness SL, et al. Usher syndrome $1 \mathrm{D}$ and nonsyndromic autosomal recessive deafness DFNB12 are caused by allelic mutations of the novel cadherin-like gene CDH23. Am J Hum Genet. 2001 Jan;68(1):26-37.

11. Ahmed ZM, Riazuddin S, Bernstein SL, Ahmed Z, Khan $S$, Griffith AJ, et al. Mutations of the protocadherin gene PCDH15 cause Usher syndrome type 1F. Am J Hum Genet. 2001 Jul;69(1):25-34.

12. Weil D, El-Amraoui A, Masmoudi S, Mustapha M, Kikkawa Y, Laine S, et al. Usher syndrome type I G (USH1G) is caused by mutations in the gene encoding SANS, a protein that associates with the USH1C protein, harmonin. Hum Mol Genet. 2003 Mar 1;12(5):463-71.

13. Riazuddin S, Belyantseva IA, Giese AP, Lee K, Indzhykulian AA, Nandamuri SP, et al. Alterations of the CIB2 calciumand integrin-binding protein cause Usher syndrome type 1J and nonsyndromic deafness DFNB48. Nat Genet. 2012 Nov;44(11):1265-71.

14. Eudy JD, Weston MD, Yao S, Hoover DM, Rehm HL, Ma-Edmonds M, et al. Mutation of a gene encoding a protein with extracellular matrix motifs in Usher syndrome type IIa. Science. 1998 Jun 12;280(5370):1753-7.

15. Hmani M, Ghorbel A, Boulila-Elgaied A, Ben Zina Z, Kammoun W, Drira M, et al. A novel locus for Usher syndrome type II, USH2B, maps to chromosome 3 at p23-24.2. Eur J Hum Genet. 1999 Apr;7(3):363-7. 
16. Weston MD, Eudy JD, Fujita S, Yao S, Usami S, Cremers C, et al. Genomic structure and identification of novel mutations in usherin, the gene responsible for Usher syndrome type IIa. Am J Hum Genet. 2000 Apr;66(4):1199-210.

17. Weston MD, Luijendijk MW, Humphrey KD, Moller C, Kimberling WJ. Mutations in the VLGR1 gene implicate Gprotein signaling in the pathogenesis of Usher syndrome type II. Am J Hum Genet. 2004 Feb;74(2):357-66.

18. Yang J, Wang L, Song H, Sokolov M. Current understanding of usher syndrome type II. Front Biosci (Landmark Ed). 2012; 17:1165-83.

19. Kimberling WJ, Weston MD, Moller C, Davenport SL, Shugart YY, Priluck IA, et al. Localization of Usher syndrome type II to chromosome 1q. Genomics. 1990 Jun;7(2):245-9.

20. Joensuu T, Hamalainen R, Yuan B, Johnson C, Tegelberg S, Gasparini $\mathrm{P}$, et al. Mutations in a novel gene with transmembrane domains underlie Usher syndrome type 3. Am J Hum Genet. 2001 Oct;69(4):673-84.

21. Adato A, Vreugde S, Joensuu T, Avidan N, Hamalainen R, Belenkiy $\mathrm{O}$, et al. USH3A transcripts encode clarin-1, a fourtransmembrane-domain protein with a possible role in sensory synapses. Eur J Hum Genet. 2002 Jun;10(6):339-50.

22. Hope CI, Bundey S, Proops D, Fielder AR. Usher syndrome in the city of Birmingham--prevalence and clinical classification. Br J Ophthalmol. 1997 Jan;81(1):46-53.

23. Rosenberg T, Haim M, Hauch AM, Parving A. The prevalence of Usher syndrome and other retinal dystrophy-hearing impairment associations. Clin Genet. 1997 May;51(5):314-21.

24. Ouyang XM, Hejtmancik JF, Jacobson SG, Li AR, Du LL, Angeli $S$, et al. Mutational spectrum in Usher syndrome type II. Clin Genet. 2004 Apr;65(4):288-93.

25. Pieke-Dahl S, van Aarem A, Dobin A, Cremers CW, Kimberling WJ. Genetic heterogeneity of Usher syndrome type II in a Dutch population. J Med Genet. 1996 Sep;33(9):753-7.

26. van Wijk E, Pennings RJ, te Brinke $H$, Claassen A, Yntema $\mathrm{HG}$, Hoefsloot LH, et al. Identification of 51 novel exons of the Usher syndrome type $2 \mathrm{~A}$ (USH2A) gene that encode multiple conserved functional domains and that are mutated in patients with Usher syndrome type II. Am J Hum Genet. 2004 Apr;74(4):738-44.

27. Beneyto MM, Cuevas JM, Millan JM, Espinos C, Mateu E, Gonzalez-Cabo P, et al. Prevalence of 2314delG mutation in Spanish patients with Usher syndrome type II (USH2). Ophthalmic Genet. 2000 Jun;21(2):123-8.

28. Dreyer B, Tranebjaerg L, Rosenberg T, Weston MD, Kimberling WJ, Nilssen O. Identification of novel USH2A mutations: implications for the structure of USH2A protein. Eur J Hum Genet. 2000 Jul;8(7):500-6.

29. Leroy BP, Aragon-Martin JA, Weston MD, Bessant DA, Willis $\mathrm{C}$, Webster AR, et al. Spectrum of mutations in USH2A in
British patients with Usher syndrome type II. Exp Eye Res. 2001 May;72(5):503-9.

30. Bernal S, Ayuso C, Antinolo G, Gimenez A, Borrego S, Trujillo MJ, et al. Mutations in USH2A in Spanish patients with autosomal recessive retinitis pigmentosa: high prevalence and phenotypic variation. J Med Genet. 2003 Jan;40(1):e8.

31. Lopez G, Gelvez NY, Tamayo M. [Mutational frequencies in usherin(USH2A gene) in 26 Colombian individuals with Usher syndrome type II]. Biomedica. 2011 Mar;31(1):82-90.

32. Liu XZ, Hope C, Liang CY, Zou JM, Xu LR, Cole T, et al. A mutation (2314delG) in the Usher syndrome type IIA gene: high prevalence and phenotypic variation. Am J Hum Genet. 1999 Apr;64(4):1221-5.

33. Aller E, Larrieu L, Jaijo T, Baux D, Espinos C, GonzalezCandelas F, et al. The USH2A c.2299delG mutation: dating its common origin in a Southern European population. Eur J Hum Genet. 2010 Jul;18(7):788-93.

34. Najera C, Beneyto M, Blanca J, Aller E, Fontcuberta A, Millan JM, et al. Mutations in myosin VIIA (MYO7A) and usherin (USH2A) in Spanish patients with Usher syndrome types I and II, respectively. Hum Mutat. 2002 Jul;20(1):76-7.

35. Rivolta C, Sweklo EA, Berson EL, Dryja TP. Missense mutation in the USH2A gene: association with recessive retinitis pigmentosa without hearing loss. Am J Hum Genet. 2000 Jun;66(6):1975-8.

36. Aller E, Najera C, Millan JM, Oltra JS, Perez-Garrigues H, Vilela C, et al. Genetic analysis of 2299delG and C759F mutations (USH2A) in patients with visual and/or auditory impairments. Eur J Hum Genet. 2004 May;12(5):407-10.

37. Garcia-Garcia G, Aparisi MJ, Jaijo T, Rodrigo R, Leon AM, Avila-Fernandez A, et al. Mutational screening of the USH2A gene in Spanish USH patients reveals 23 novel pathogenic mutations. Orphanet J Rare Dis. 2011;6:65.

38. Nakanishi H, Ohtsubo M, Iwasaki S, Hotta Y, Usami S, Mizuta K, et al. Novel USH2A mutations in Japanese Usher syndrome type 2 patients: marked differences in the mutation spectrum between the Japanese and other populations. J Hum Genet. 2011 Jul;56(7):484-90.

39. Zhao Y, Hosono K, Suto K, Ishigami C, Arai Y, Hikoya A, et al. The first USH2A mutation analysis of Japanese autosomal recessive retinitis pigmentosa patients: a totally different mutation profile with the lack of frequent mutations found in Caucasian patients. J Hum Genet. 2014 Sep;59(9):521-8.

40. Vastinsalo H, Jalkanen R, Bergmann C, Neuhaus C, Kleemola L, Jauhola L, et al. Extended mutation spectrum of Usher syndrome in Finland. Acta Ophthalmol. 2013 Jun;91(4):325-34.

41. Huang XF, Xiang P, Chen J, Xing DJ, Huang N, Min Q, et al. Targeted exome sequencing identified novel USH2A mutations in Usher syndrome families. PLoS One. 2013;8(5):e63832. 


\title{
Optimización de la Reacción en Cadena de la Polimerasa para la Detección del gen B1 de T. gondii
}

\author{
Polymerase Chain Reaction Optimization for Detection of gen B1 \\ Optimización de PCR para el gen B1 de T. gondii
}

Cortés Cortés Liliana Jazmín ${ }^{1}$, Hernández Castro Diana Carolina ${ }^{1}$, Mantilla Mónica², Medina María Isabel ${ }^{2}$, Duque Sofía ${ }^{1}$.

\section{Resumen}

Objetivo: Optimizar las condiciones de amplificación del gen B1 (35 copias en el genoma) para la detección de ADN de T.gondii en casos probables de toxoplasmosis cerebral. Materiales y métodos: Se realizó extracción de $\mathrm{ADN}$ a partir de exudado peritoneal de ratones inoculados con la cepa RH de T. gondii obteniendo $17 \mathrm{~mL}$ con una concentración inicial de $1 \times 10^{7}$ parásitos $/ \mathrm{mL}$. Se optimizaron las condiciones de PCR del gen B1. Resultados: Se obtuvo amplificación de un fragmento de $132 \mathrm{pb}$ a partir de ADN obtenido de diluciones seriadas desde $1 \times 10^{6}$ a $1 \times 10^{-1}$ parásitos por $\mathrm{mL}$, estableciéndose un límite de detección de 1 taquizoíto de T. gondii.

Palabras clave: toxoplasmosis, optimización, reacción en cadena de la polimerasa, gen B1, detección.

\section{Abstract}

Objective: This study aimed to optimize the conditions B1 gene amplification (35 copies in the genome) for the detection of. $T$ gondii DNA in probable cases of cerebral toxoplasmosis. Materials $y$ methods: DNA extraction was performed from the peritoneal exudate of mice inoculated with the RH strain of $T$. gondii obtaining an initial $17 \mathrm{~mL}$ to $1 \times 10^{7}$ parasites $/ \mathrm{mL}$. PCR conditions yielding B1 gene amplification of a fragment of $132 \mathrm{bp}$ DNA obtained from serial dilutions from $1 \times 10^{6}$ to $1 \times 10^{-1}$ parasites per $\mathrm{ml}$ were optimized, setting a limit of detection of $1 \mathrm{~T}$. gondii tachyzoite. Resulted: B1 gene amplification of a fragment of 132 bp DNA obtained from serial dilutions from $1 \times 10^{6}$ to $1 \times 10^{-1}$ parasites per $\mathrm{mL}$ were optimized, setting a limit of detection of 1 T. gondii tachyzoite.

Keywords: toxoplasmosis, optimization, chain reaction polymerase, B1 gene detection.

${ }^{1}$ Instituto Nacional de Salud. Dirección de Investigación en Salud Pública, Subdirección de

Investigación Científica y Tecnológica, Grupo de Parasitología.

2 Hospital Simón Bolívar E.S.E.

Correspondencia: jcortes@ins.gov.co. 


\section{Introducción}

La toxoplasmosis es una zoonosis mundial cuyos huéspedes definitivos son los felinos y como huéspedes intermediarios se encuentran el hombre y la mayoría de animales de sangre caliente (1). En el humano, la mayoría de las infecciones por T. gondii son subclínicas, sin embargo una infección severa puede ocurrir en los pacientes inmunocomprometidos dentro de los que se encuentran los pacientes con VIH/SIDA y con tumores malignos (2). En los pacientes con SIDA, a pesar de la terapia antirretroviral, la toxoplasmosis cerebral (TC) es la infección oportunista más frecuente (3).

La optimización de las condiciones de amplificación por PCR para el gen B1 de T. gondii, permitirá ser la base para realizar el diagnóstico molecular en pacientes con probable toxoplasmosis cerebral a través de la técnica de PCR en muestras biológicas de Liquido Cefalo Raquídeo (LCR) y sangre, ya que en Colombia, el diagnóstico de toxoplasmosis cerebral se realiza presuntivamente mediante hallazgos clínicos, radiológicos, serológicos y la respuesta a la terapia anti-Toxoplasma (4).

Es importante tener en cuenta que la mayoría de microorganismos que producen cuadros neurológicos en pacientes inmunosuprimidos (T. cruzi, Cryptococcus neoformans, Esptein-Barr entre otros) también causan sintomatología y se evidencian hallazgos radiológicos similares a los que se presentan en la TC, por lo que se resalta la importancia de realizar la PCR en LCR y sangre para hacer diagnóstico diferencial de TC y formular el tratamiento adecuado para el paciente (5).

\section{Materiales y métodos \\ Mantenimiento de la cepa RH de T. gondii en modelo animal}

Se realizó el mantenimiento de la cepa de referencia RH de T. gondii (ATCC 50174) a través de pases sucesivos en ratones de la cepa ICR-CD1, machos y hembras, de 18 a 20 días de edad, mediante la inoculación intraperitoneal con $0,3 \mathrm{~mL}$ de taquizoitos a una concentración de $1 \times 10^{6}$ parásitos $/ \mathrm{mL}$.

Se mantuvieron en el bioterio los animales inoculados, debidamente identificados, siguiendo las normas CICUAL (6). Se revisaron diariamente para detectar cambios en el comportamiento como letargia e inactividad y cambios físicos como irritación ocular, hepato o esplenomegalia, piloerección que evidenciaron la infección por T. gondii dándose así punto final al experimento y mediante eutanasia de los ratones en cámara de $\mathrm{CO}_{2}$ recolectar, del exudado de éstos, un mínimo $10^{6}$ taquizoítos del parásito/mL.

\section{Extracción de ADN parasitario}

Se realizó la extracción del ADN parasitario a partir del exudado peritoneal v/v con Clorhidrato de Guanidina, con una concentración de $1 \times 10^{6}$ parásitos/mL, con el kit PureLinkTM Genomic DNA Kits, siguiendo las instrucciones del fabricante así: Se resuspendieron $200 \mu \mathrm{l}$ de cada una de las muestras (cepa de referencia RH de T. gondii) en $200 \mu \mathrm{l}$ de Solución Salina estéril, se adicionaron $20 \mu \mathrm{l}$ de Proteinasa K a la muestra, no se adicionó RNAsa A a la muestra, porque puede producir inhibición, se mezcló suavemente con vórtex, se incubó por 2 minutos a temperatura ambiente, se adicionaron $200 \mu \mathrm{l}$ de PureLinkTM Genomic Lysis/Binding Buffer y se mezcló suavemente con vórtex. Se incubó a $55^{\circ} \mathrm{C}$ por 10 minutos para promover la digestión proteica. Se adicionaron $200 \mu \mathrm{l}$ de etanol (96-100\%) al lisado. Se mezcló suavemente con vórtex por 5 segundos.

Unión del $A D N$. Se tomó una columna PureLinkTM Spin Column del paquete. Se adicionó el lisado obtenido en el paso anterior. Se centrifugó la columna a $10.000 \mathrm{~g}$ por 3 minutos a temperatura ambiente. Se descartó el tubo colector y se transfirió la columna a un nuevo tubo colector.

Lavado de ADN. Se adicionaron $500 \mu \mathrm{l}$ de Buffer de Lavado 1 a la columna. Se centrifugó la columna 
a 10.000 g por 3 minutos a temperatura ambiente. Se descartó el tubo colector y se transfirió la columna a un nuevo tubo colector. Se adicionaron $500 \mu \mathrm{l}$ de Buffer de Lavado 2 a la columna. Se centrifugó la columna a máxima velocidad por 3 minutos a temperatura ambiente.

Elución del $A D N$. Se transfirió la columna a un tubo eppendorf estéril de $1,5 \mathrm{ml}$. Se adicionaron $100 \mu \mathrm{l}$ de buffer de elución del kit. Se incubó a temperatura ambiente por 2 minutos. Se centrifugó la columna a máxima velocidad por 3 minutos a temperatura ambiente. Se descartó la columna, el tubo colector es el que contiene el ADN. Se almacenó el $\mathrm{ADN}$ a $-40^{\circ} \mathrm{C}$.

Cuantificación de ADN. Se realizó la cuantificación del ADN obtenido mediante espectrofotómetro NanoDrop ${ }^{\text {тм }} 2000$ utilizando $2 \mu$ l de la muestra conservando la tensión superficial y la estática entre dos fibras ópticas sin la necesidad de utilizar diluciones.

\section{Optimización de la técnica de PCR.}

Se realizaron los análisis moleculares con el ADN del parásito, extraído a partir de exudado peritoneal de ratones inoculados cepa ICR-CD1.

Se utilizaron los oligonucleótidos reportados por Costa JM y Bretagne S (7) y tomando como base las condiciones de amplificación para el gen $\mathrm{B} 1$ informadas por Reischi y col. (8), se optimizaron las temperaturas óptimas de hibridación (Tms) de los oligonucleótidos, el tamaño del fragmento esperado, el tiempo de desnaturalización como el de extensión, el número de ciclos y el tiempo del ciclo de extensión final.

\section{Determinación de la sensibilidad de la técnica de PCR para la detección del gen B1 de T. gondii}

Se determinó la sensibilidad para la detección del gen $\mathrm{B} 1$ del parásito con diferentes concentraciones de ADN de la cepa RH de T. gondii. Para establecer la concentración de ADN obtenida se realizó la cuantificación por espectrofotometría con el NA-
NODROP 2000 y a partir de esta concentración se prepararon diluciones de $1 \times 10^{6}$ parásitos $/ \mathrm{mL}$, $1 \times 10^{5}$ parásitos $/ \mathrm{mL}, 1 \times 10^{4}$ parásitos $/ \mathrm{mL}, 1 \times 10^{3}$ parásitos $/ \mathrm{ml}, 1 \times 10^{2}$ parásitos $/ \mathrm{ml}, 1 \times 10^{1}$ parásitos/ $\mathrm{mL}, 1 \times 10^{0}$ parásitos $/ \mathrm{ml}, 1 \times 10^{-1}$ parásitos $/ \mathrm{mL}$; para determinar la mínima cantidad de ADN detectada por los oligonucleótidos del gen B1.

\section{Ensayos de exclusividad de la técnica de PCR} para la detección del gen B1 de T. gondii

Se determinó la especificidad de los oligonucleótidos mediante la amplificación de ADN de otros microorganismos como Plasmodium falciparum, Trypanosoma cruzi y Leishmania braziliensis.

\section{Resultados}

Luego de la extracción de ADN, se realizó la respectiva cuantificación obteniéndose una concentración promedio de ADN parasitaria de $28.5 \mathrm{ng} / \mathrm{mL}$. La mezcla de reacción para la amplificación del gen B1 fue optimizada así: GoTaq Hot Start Green Master Mix 1X, oligonucleótidos directo y reverso 4 pM y $5 \mu \mathrm{ADN}$ para un volumen final de 25 $\mu$ l. Las condiciones de amplificación optimizadas para el gen B1 se encuentran en la Tabla 1 .

Tabla 1. Condiciones de amplificación para el gen B1 de $T$. gondii

\section{CONDICIONES GEN B1}

Ciclos 50 ciclos

Acondicionamiento $95^{\circ} \mathrm{C} / 10$ minutos

Desnaturalización $95^{\circ} \mathrm{C} / 1$ minuto

Anillamiento $56^{\circ} \mathrm{C} / 1$ minuto

Extensión $72^{\circ} \mathrm{C} / 1$ minuto

Extensión final $72^{\circ} \mathrm{C} / 10$ minutos

La visualización del producto de amplificación, se realizó mediante una electroforesis en gel de agarosa (2\%), teńido con Gel Red.

En cuanto al límite de detección de PCR para detección de $T$. gondii la mínima concentración de ADN detectada por los oligonucleótidos del gen B1, corresponde a la dilución $10^{\circ}$, es decir a 1 taquizoíto de $T$. gondii. Figura 1. 
Figura 1. Límite de detección de PCR para detección de T. gondii y especificidad mediante amplificación de ADN obtenido de otros microorganismos como P. falciparum y T. cruzi.

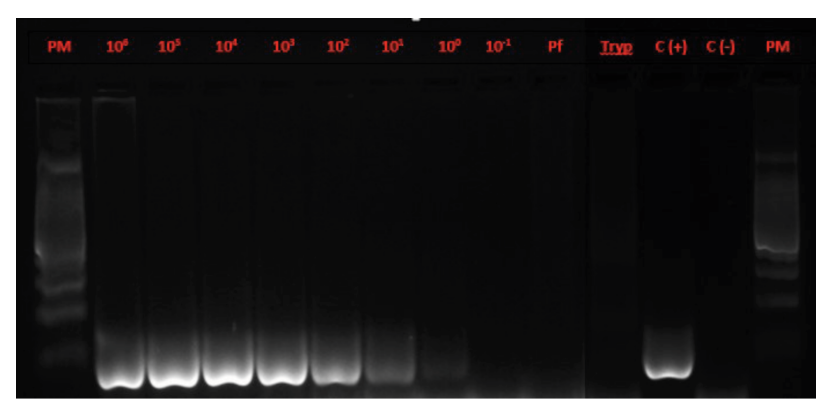

Mediante los ensayos de exclusividad de la técnica de PCR para la detección del gen B1 de T. gondii se determinó la especificidad de los oligonucleótidos mediante la amplificación de ADN obtenido de otros microorganismos como $P$. falciparum, $T$. cruzi y L. braziliensis. Al amplificar el ADN de estos organismos con los oligonucleótidos para el gen $\mathrm{B} 1$ bajo las mismas condiciones de amplificación no se observó ninguna reacción cruzada. Figuras 1 y 2.

Figura 2. Especificidad de PCR para detección de T. gondii mediante la amplificación de diferentes diluciones de ADN de $L$. braziliensis.

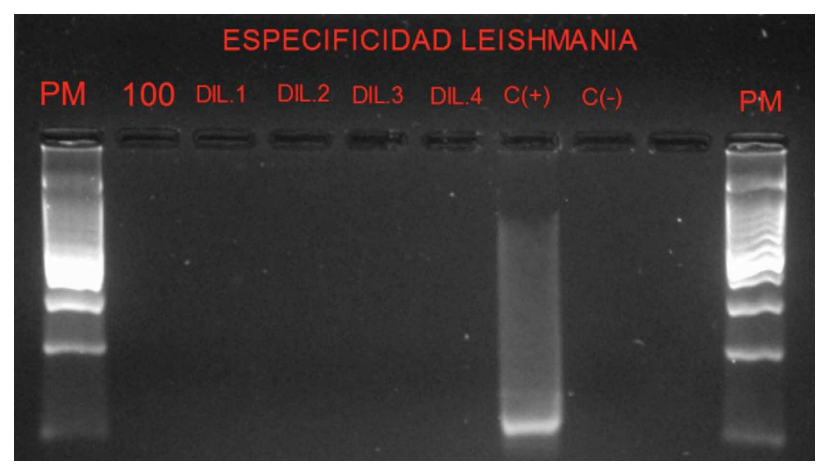

\section{Discusión}

La utilidad de los métodos biológicos moleculares ha sido investigada como herramienta complementaria del manejo clínico de afecciones como la toxoplasmosis cerebral. El uso de la técnica de PCR ha sido ampliamente recomendado para amplificar y posteriormente detectar el ADN de microorganismos en una variedad de líquidos y tejidos, particularmente aquellos que son difíciles para su detección directa por la complejidad de su aislamiento a partir de cultivos (9) o por el volumen reducido de la muestra, en cuyo caso ha demostrado ser muy útil (10).

La técnica de PCR para la detección del gen B1 de $T$. gondii, que se ha descrito puede ser utilizada para la confirmación diagnóstica de toxoplasmosis cerebral en muestras de sangre y LCR (11) la cual podría implementarse en los Laboratorios clínicos de Instituciones de Salud de tercer y cuarto nivel.

A través de este trabajo se optimizaron las condiciones de una técnica de PCR que permite la detección de $T$. gondii. Por medio de los ensayos de límite de detección se pudo establecer que esta técnica es capaz de detectar mínimo un taquizoíto presente en la muestra examinada y mediante los ensayos de exclusión se determinó que no se presentan reacciones cruzadas con otro patógenos (como Virus Epstein Barr, Virus JC, T. cruzi o Criptococcus neoformans entre otros) (12) que producen alteraciones cerebrales en pacientes inmunosuprimidos, mostrando que el gen B1 es altamente específico para T. gondii (13). 


\section{Referencias}

1. Priest J, Moss D, Arnold B, Hamlin K, Jones C, Lammie P. Seroepidemiology of Toxoplasma in a coastal region of Haiti: multiplex bead assay detection of immunoglobulin $\mathrm{G}$ antibodies that recognize the SAG2A antigen. Epidemiol Infect. 2015; 143(3): 618-30. doi: 10.1017/S0950268814001216.

2. Ahmadpour E, Daryani A, Sharif M, Sarvi S, Aarabi M, Mizani A, et. al. Toxoplasmosis in immunocompromised patients in Iran: a systematic review and meta-analysis.J Infect Dev Ctries. 2014; 15(12):1503-10. doi: 10.3855/jidc. 4796.

3. Meira C, Pereira-Chioccola V, Vidal J, de Mattos C, Motoie G, Costa-Silva TA, et. al. Cerebral and ocular toxoplasmosis related with IFN- $\gamma$, TNF- $\alpha$, and IL-10 levels. Front Microbiol. 2014; 5:492. doi: 10.3389/fmicb.2014.00492. eCollection 2014.

4. Tonini R, Vidal J, Vera L. Molecular diagnosis of cerebral toxoplasmosis: comparing markers that determine Toxoplasma gondii by PCR in peripheral blood from HIV-infected patients The Brazilian Journal of Infectious Diseases. 2010; 14(4): 346-50.

5. Anselmo L, Vilar F, Lima J, Yamamoto A, Bollela V, Takayanagui $\mathrm{O}$. Usefulness and limitations of polymerase chain reaction in the etiologic diagnosis of neurotoxoplasmosis in immunocompromised patients. J Neurol Sci. 2014; 346 (1-2):231-4. doi: $10.1016 /$ j.jns.2014.08.034.

6. Resolución 458 de 2011, Instituto Nacional de Salud.

7. Bretagne S, Costa JM, Vidaud M, Van Nhieu JT, Feith J. Detection of Toxoplasma gondii by Competitive DNA Amplification of Bronchoalveolar Lavage Samples. JID 1993;168 (6): 1585-88.
8. Reishi U, Bretagne S, Kruger D, Ernault P, Costa JM: Comparison of two targets for the diagnosis of Toxoplasmosis by real-time PCR using fluorescence resonance energy transfer hybridization probes. BMC Infect Dis. 2003; 3: 7 doi 10.1186/1471-2334-37.

9. Asgari Q, Keshavarz H, Shojaee S, Motazedian M, Mohebali M, Miri R, et al. In Vitro and In Vivo Potential of RH Strain of Toxoplasma gondii (Type I) in Tissue Cyst Forming. Iran J Parasitol. 2013; 8(3):367-75.

10. Taravati P, Lam D, Van Gelder R. Role of molecular diagnostics in ocular microbiology. Curr Ophthalmol Rep. 2013;1(4). doi: 10.1007/s40135-013-0025-1.

11. Bourdin C, Busse A, Kouamou E, Touafek F, Bodaghi B, Le Hoang P, Mazier, et al. PCR-based detection of Toxoplasma gondii DNA in blood and ocular samples for diagnosis of ocular toxoplasmosis. J Clin Microbiol. 2014; 52(11):3987-91. doi: 10.1128/JCM.01793-14.

12. Xiao J, Gao G, Li Y, Zhang W, Tian Y, et al. Spectrums of Opportunistic Infections and Malignancies in HIV-Infected Patients in Tertiary Care Hospital, China. PLoS ONE. 2013; 8(10): e75915. doi:10.1371/journal.pone.0075915.

13. Cortés LJ, Duque S, López MC, Moncada D, Molina D, Gómez-Marín JE, Gunturiz ML. Gene polymorphisms in the dihydrofolate reductase $(d h f r)$ and dihydropteroate synthase (dhps) genes and structural modelling of the dhps gene in Colombian isolates of Toxoplasma gondii. Biomedica. 2014; 34(4):556-66. doi: 10.1590/S0120-41572014000400008. 